\title{
AUTOMATIC ROI EXTRACTION IN NOISY MEDICAL IMAGES
}

\author{
S. Renukalatha ${ }^{1}$ and K.V. Suresh ${ }^{2}$ \\ ${ }^{1}$ Department of Computer Science and Engineering, Sri Siddhartha Institute of Technology, India \\ ${ }^{2}$ Department of Electronics and Communication Engineering, Siddaganga Institute of Technology, India
}

\begin{abstract}
Accurate segmentation of medical images is pivotal in medical image analysis as it favors the detection and quantification of abnormalities present in human anatomical structures. Since medical images are complex and sometimes noisy, effective extraction of the regions of abnormalities is a tedious process. Many semi-automatic segmentation algorithms with appreciable segmentation accuracy do exist in literature. However, these techniques are iterative, computationally expensive, involve human intervention demanding initial parameter settings and moreover, each one of them is specific to a particular modality. In addition, presence of noise further degrades the quality of the processed image. There is no general algorithm to extract the key regions from all types of noisy medical images. This paper proposes an automatic Region of Interest (ROI) extraction algorithm to detect the important regions in noisy medical images of different modalities using statistical moments. The proposed approach estimates an optimal threshold value automatically using statistical moments through histogram decomposition technique. Initially, the medical image database is preprocessed followed by $\mathrm{ROI}$ extraction and the performance of the proposed approach is compared with other techniques to verify its robustness.
\end{abstract}

Keywords:

Histogram, Optimal Threshold, Moments, ROI, Medical Imaging Modalities

\section{INTRODUCTION}

There is no universal algorithm for segmentation of every medical image. It is very difficult to devise a ROI method which adapts to medical images of different modalities. Each imaging system has its own specific limitations. However, accurate segmentation of medical images is a key step in medical image analysis for proper diagnosis by a medical expert. Automatic ROI detection in medical images is a difficult task as medical images are complex in nature. Further, the success rate of a detection algorithm is affected due to: presence of noise, artifacts caused due to insufficient illumination and overlapping pixel intensities of anatomical structures. Literature records several algorithms in the field of medical image segmentation [1,2]. Among them, threshold based segmentation procedure is found to be very simple, robust, accurate and has less computational time complexity [3]. However, determining the best threshold automatically for accurate segmentation is a tedious procedure for complex and noisy medical image database. Techniques for automatic determination of threshold value can be classified as: determination of global threshold value or general threshold value and determination of local threshold value [4]. Global thresholding computes the threshold value automatically based on statistical information of the image. Very popular global thresholding technique is Otsu's approach [5]. Other global thresholding techniques in use are: Kapur, Kittler and Triangle methods. The main drawbacks of these methods are: computational complexity, consume more time and efficiency varies with the quality of the image. These methods are efficient and suitable only for medical images of high resolution and good contrast and they do not perform well for segmentation of images with multiple objects each having distinct gray level value varying over a band of values [6]. Whereas, local thresholding or adaptive thresholding approach determines the threshold value based on local statistics and threshold value found is locally optimal for small areas [7]. But these techniques are iterative based and time complexity is high. Hence, there is a scope for detection of a best optimal threshold value for automatic extraction of ROIs from inconsistent medical images. In such cases, a more appropriate method to choose a threshold value is histogram based approach. Histogram of an image may be unimodal, bimodal and multimodal. In such cases, the valley point between the two modes is the threshold value as shown in Fig.1(b).

Histogram-based thresholding is one of the common methods used in image thresholding and segmentation [8] It is simple because it is easier to visualize and accurate enough to differentiate the target from the background. Thus, many methods used this ability to calculate the optimal threshold value [9]. However, most of the medical image histograms are more complex, with many peaks and not clear valleys, and the histogram distribution is always not distinct as shown in Fig.1(c) and it is not always easy to find the optimal threshold value [10]. To address this problem, automatic multi-thresholding techniques [11,12] are employed to achieve the segmentation accuracy. In [13], multi-thresholding technique using regularization approach is proposed. Initially, histogram is smoothed and later peaks and valley points are extracted using predefined score function. In another approach proposed by [14] obtains multi-scale information of the histogram by convolving it with second order derivative of the Gaussian filter to obtain the best possible threshold values. However, major problem in multithresholding techniques is finding the optimal threshold value due to the fact that number of objects are always unknown due to the presence of overlapping tissues, noise and other artifacts. Hence, accurate selection of a threshold value from such images is very crucial [15].

In addition, presence of noise in medical images degrades visual quality of an image and spoils important information required for accurate diagnosis. Removal of these noise components from the image without destroying the useful information is highly challenging. Hence, noise removal is often necessary and the first step in the medical image analysis process. The image acquisition system used to generate medical images usually produce some noise depending on the type of imaging modality. To achieve the finest possible details required for an accurate diagnosis of diseases it is necessary that medical images to be sharp, clear and free of noise and artifacts. In this paper, the medical images of different modalities prone to various types of 
noise components along with approximation and reduction is addressed in preprocessing stage. Once these images are preprocessed, their histograms must be smoothed to get clear valley and peak points to obtain precise threshold value for accurate segmentation. According to the assumption in [16], smoothed grey-level histogram is similar to mixture of Gaussian distributions. That is, each region in an image is Gaussian-like distribution possessing different statistical moments such as mean, variance and probabilities. In general, many of these thresholding methods produced good segmentation results. However, some of the approaches demand a high processing time, which make these methods impractical when the number of thresholds used exceed three for multi-class segmentation [17]. Other approaches are not fully automatic, sensitive to the uniformity of the histogram and demand a prior knowledge about the number of classes in the histogram. To overcome these difficulties, an automatic thresholding approach which determines the optimal threshold value without using the prior knowledge of number of objects is proposed. The proposed method involves preprocessing of medical images in the first level to achieve histogram smoothing through denoising and contrast enhancement to get local minimum points as initial thresholds. Rest of the paper is organized as follows.

In Section 2, the mathematical background of threshold calculation by histogram decomposition method is briefly described. Section 3 lists various performance metrics used for evaluation. Section 4 gives the complete flowchart of the proposed methodology. Experimental results are given in section 5. Section 6 concludes the paper.

\section{MATHEMATICAL BACKGROUND: DENOISING AND HISTOGRAM ANALYSIS}

As medical images are of varying grey level complexities and sometimes noisy, it is difficult to devise a single ROI algorithm using a simple histogram thresholding. It is very tough to find the exact threshold value as image histograms of many medical images do not possess clear valley points. However, it would be possible get clear valley points in image histograms through preprocessing methods such as: image denoising and image enhancement. Hence, the proposed method combines preprocessing methods with histogram decomposition to achieve accurate ROI extraction of abnormalities. This section describes the mathematical basis of histogram analysis procedure employed on preprocessed medical images.

\subsection{MEDICAL IMAGE DENOISING AND ITS APPROXIMATION}

Presence of noise degrades the quality of medical images and lead to improper disease diagnosis. Many researchers have proposed denoising techniques to reduce noise in medical images [18]. The image acquisition system used to generate the medical images usually produce some noise depending on the type of imaging modality. Usually, MRI images are corrupted by Rician noise, X-ray images suffer from Poisson noise, CT images are associated with Poisson- Gaussian noise and Ultrasound images are more prone to speckle noise. Choice of denoising filter is based on the type of medical image selected for analysis.

\subsubsection{MRI Image Denoising:}

Noise in MR images obeys a Rician distribution [19]. Many denoising filters for Rician noise removal have been reported in the literature. Probability density function (PDF) of Rician noise [20] is given by Eq.(1).

$$
\begin{gathered}
(q \mid t, \sigma)=\frac{t}{\sigma^{2}} \exp \left(-\frac{t^{2}+q^{2}}{2 \sigma^{2}}\right) I_{0}\left(\frac{t q}{\sigma^{2}}\right) \\
q=\sqrt{\left(t+n_{r}\right)^{2}+n_{i}^{2}}
\end{gathered}
$$

where, $t$ is the true signal (pixel) intensity, $q$ is the observed image pixel intensity, $\sigma$ is the standard deviation of the Gaussian noise in the real and the imaginary images, and $I_{0}$ is the zero-order modified Bessel function of the first kind. The Eq.(2) represents the magnitude image equation for MRI images. A special case of the Rician distribution is in image regions where only noise is present and $S N R=q / \sigma=0$ (e.g. in the dark background areas of an MRI where no signal is present). This special case of the Rician distribution, where $q=0$ and $I_{0}=1$ is also known as the Rayleigh distribution:

$$
f(s)_{\text {Rayleigh }}=\frac{s}{\sigma^{2}} \exp \left(-\frac{s^{2}}{2 \sigma^{2}}\right)
$$

In the image regions where the signal is present and $S N R \geq 3$, the noise distribution approximates a Gaussian distribution. Thus, the problem of Rician noise in the brain MRI is often simplified in practice by assuming the Gaussian distribution for the noise, [21].

$$
f(t)_{\text {Gauss }}=\frac{1}{\sigma \sqrt{2 \pi}} \exp \left(-\frac{(t-\mu)^{2}}{2 \sigma^{2}}\right)
$$

With reference to Eq.(3), the proposed method initially uses Gauss filter and later, edge preserving anisotropic diffusion filter to reduce Rician noise in MRI images.

\subsubsection{X-ray Image Denoising:}

Noise in X-ray images is dominated by additive Poissondistributed quantum noise [22]. Individual photon detections can be treated as independent events that follow a random temporal distribution. According to central limit theorem, which says that the sum of large number of independent quantities tends to have a Gaussian form independent of the individual measurements. As a result, photon counting is a classic Poisson process, and the number of photons $N$ measured by a given sensor element over a time interval $t$ is described by the discrete probability distribution,

$$
p_{r}(N=k)=\frac{e^{-\lambda t} \lambda t^{k}}{k !}
$$

where, $\lambda$ is the expected number of photons per unit time interval, which is proportional to the incident scene irradiance. This is a standard Poisson distribution with a rate $\lambda t$ parameter that corresponds to the expected incident photon count. The uncertainty described by this distribution is known as photon noise. For a sufficiently large number of quanta contributing per pixel, the Poisson distribution can be approximated by a Gaussian distribution. Hence, the proposed method uses a combination of Gauss filter and diffusion filter for noise reduction in X-Ray images. 


\subsubsection{CT Image Denoising:}

Poisson noise is generated due to random pattern of photons emitted by X-rays in the detector and found to be Poisson distributed as in X-rays. However, it cannot be approximated to Gaussian noise as noise in CT images is caused by low dose Xrays. According to Flourian Luiser [23], transform domain approach reduces the Poisson- Gaussian noise effectively. The proposed algorithm uses multispinning method in transform domain [24] for noise reduction which makes use of seven images in multiple versions to acquire the boundary information in transform domain. Later, noisy images are subjected to multispinning algorithm and wavelet transform is applied to all the cyclic shifted images and there by denoised images are obtained. Inverse transform reconstructs the denoised images. Further, denoised images are unshifted and averaged. This process can be depicted by the Eq.(5).

$$
\hat{d}=\frac{1}{N} \sum_{i=1}^{N} S_{u p n}\left[W T_{m}^{-1}\left\{D\left(W T_{m}\left(S_{p n}\left(\left\{D_{n}\right\}_{i}\right)\right)\right)\right\}\right]
$$

where, $\hat{d}$ is the multispinning denoised image, $d_{n}$ is noisy image, $S_{p n}$ is multispin shift, $S_{u p n}$ is multispin-unshift, $W T_{m}$ is the wavelet transform, $N$ is the number of images in the denoising process, $W T_{m}^{-1}$ is the inverse transform. This process avoids the Gibb's problem [25] through averaging and the quality of the reconstructed image improves due to the information captured in different image versions.

\subsubsection{Ultrasound (US) Image Denoising:}

Speckle noise in US images is multiplicative which displays a granular pattern due to the dispersion of the electromagnetic waves caused by the transducer. This noise degrades the fine details and limits the contrast resolution by making it difficult to detect small and low contrast lesions in Ultrasound images. The multiplicative noise model, where the noise signal gets multiplied to the original signal is given by,

$$
f_{n}(x, y)=f(x, y) * \eta(x, y)
$$

where, $f_{n}(x, y)$ is the speckle noise affected image, $f(x, y)$ is the original noise-free image and $\eta(x, y)$ is the noise component. Homomorphic filtering technique is more effective in Ultrasound image denoising [26]. In the proposed algorithm, Homomorphic filtering is employed which involves transformation of multiplicative noise into additive noise by applying logarithm function. Filter transfer function of Homomorphic process uses $3^{\text {rd }}$ order Butterworth low-pass filter. Various stages of Butterworth- Homomorphic filter are given by:

Step 1: Applying natural log on both sides of Eq.(6),

$$
\begin{aligned}
& \ln f_{n}(x, y)=\ln [f(x, y) * \eta(x, y)] \\
& \text { Let, } \ln f_{n}(x, y)=z(x, y)
\end{aligned}
$$

Step 2: $z(x, y)=\ln [f(x, y)]+\ln [\eta(x, y)]$

Step 3: Fourier transform of the above equation yields frequency domain representation,

$$
Z_{n}(u, v)=F_{i}(u, v)+F_{\eta}(u, v)
$$

Step 4: Now multiplying $Z_{n}(u, v)$ with filter transfer function $H(u, v)$,

$$
Z_{n}(u, v) H(u, v)=S(u, v)=F_{i}(u, v) H(u, v)+F_{\eta}(u, v) H(u, v)
$$

where, $H(u, v)$ is the transfer function of Butterworth low-pass filter. Preprocessed image $s(x, y)$ is obtained using inverse Fourier transform. The transfer function of the Butterworth low-pass filter of order $n$ and with cut-off frequency locus at a distance $D_{0}$ from the origin is defined by the relation:

$$
H(u, v)=\frac{1}{1+\left[\frac{D(u, v)}{D_{0}}\right]^{2 n}}
$$

\subsection{GRAY-LEVEL HISTOGRAM ANALYSIS}

Let $I$ be a $m \times n$ gray-scale medical image with $L$ gray levels and $G$ be the gray value of pixel $I(x, y)$ with $x=1,2, \ldots, p$ and $y=$ $1,2, \ldots, q$. Then, the gray-level histogram $H$ of image $I$ is of the form $H=\{H(j), j \in[1, G]\}$. Generally, there exists a number of peaks (distributions) in the histogram, based on the complexity of gray level distribution in an image. Each distribution in the histogram will map to an object in the image. For any gray-level histogram with $\mathrm{n}$ distributions, the histogram-thresholding technique is to automatically determine the optimal threshold value. For a complex medical image, it is assumed that the observation comes from a mixture of $n+1$ Gaussian distributions, $f$, having respective means and variances $\left(m_{1}, \sigma_{1}^{2}\right), \ldots,\left(m_{n+1}, \sigma_{n+1}^{2}\right)$ with respective proportions, $P_{1}, \ldots P_{n+1}$. Therefore, the mixture of distributions reflected in the histogram will be of the form,

$$
f(k)=\sum_{i=1}^{n+1} \frac{P_{i}}{\sqrt{2 \pi \sigma_{i}}} \exp \left\{-\frac{1}{2}\left(\frac{k-m_{i}}{\sigma_{i}}\right)^{2}\right\} .
$$

The main objective is to find the parameters, i.e., means, variances and proportions to satisfy the minimization, $\min (|f-H|)$. Later, by using these parameters, the optimal threshold value can be determined to make suitable segmentation. If the clusters in the histogram are over-lapping, it is difficult to find the precise valley point and hence the threshold value. To overcome this, gray level histogram is decomposed into several non-overlapping distributions first and later other parameters are estimated [27]. However, as some medical images are complex, the histogram distribution is always not distinct as shown in Fig.1(c). Hence, a histogram smoothing process, Fig.1(b) and Fig.1(d) is necessary before performing the decomposition process.

Then, the genuine local minimums are extracted and used as initial threshold values for further parameter estimation. For every cluster in the histogram, there exists only one optimal interval near the cluster center with the smallest absolute skewness value, for parameter estimation. The skewness is a measure of the asymmetry of the probability distribution of a real valued random variable. By using this optimal estimation interval, statistical moments such as: mean, variance, probabilities and skewness corresponding to each cluster can be predicted without iterative parameter refinement. This reduces the algorithmic time complexity. Later, based on maximum- likelihood decision theory, optimal threshold values are estimated.

In the proposed approach, histogram smoothing is carried-out through an image enhancement technique - Contrast Limited Adaptive Histogram Equalization (CLAHE) [28] which operates 
on small regions in the image called tiles, rather than the entire image. Each tile's contrast is enhanced using cumulative distribution function (CDF), so that the histogram of the output region approximately matches the histogram specified by the distribution parameter. The neighboring tiles are then combined using bilinear interpolation to eliminate artificially induced boundaries. The contrast, especially in homogeneous areas, can be limited to avoid amplifying any noise that might be present in the image. Let $I$ be a given image of size $m \times n$ matrix with pixel intensities ranging from 0 to $L-1$. Here, $L$ is the number of possible intensity values, often 256. Let, $h$ denote the normalized histogram of $I$ with a bin for each possible intensity.
Original Histogram

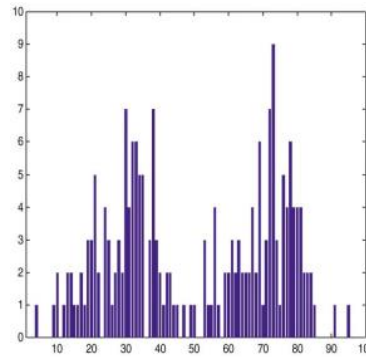

(a)

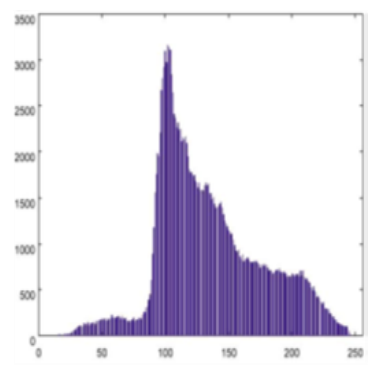

(c)

Histogram with non-distinct peaks and valley points

\section{Histogram after smoothing}

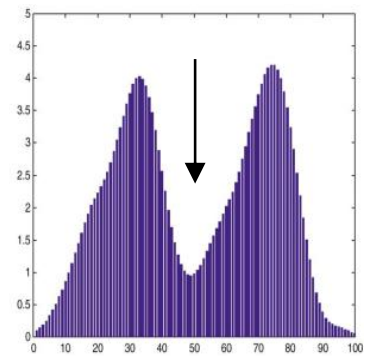

(b)

Histogram with two distinct peaks and one valley. Threshold value is 50

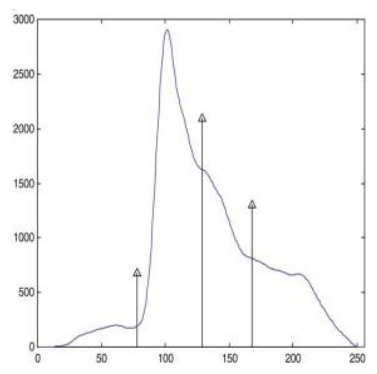

(d)

Smoothed histogram.

Threshold values are: 80 , 130 and 170
Fig.1. Example showing the image histograms of two images before and after smoothing

The probability of an occurrence of a pixel of level $i$ in the image is,

$$
\begin{gathered}
h_{x}(i)=h(x=i)=\frac{n^{i}}{n}, 0 \leq i \leq L \\
h_{x}=\frac{\text { number of pixels with intensity } n}{\text { total number of pixels }}
\end{gathered}
$$

where, $n=0,1, \ldots, L-1$.

Cumulative distribution function for $h_{x}$ is given by,

$$
c d f_{x}(i)=\sum_{j=0}^{i} h_{x}(j) .
$$

The histogram equalized image $H_{e}$ will be defined by,

$$
H_{e}=\text { floor }\left((L-1) \sum_{n=0}^{f_{i, j}} h_{x}\right)
$$

where, floor () rounds down to the nearest integer. This is equivalent to transforming the pixel intensities, $n$, of $I$ by the function,

$$
T(n)=\text { floor }\left((L-1) \sum_{n=0}^{k} h_{n}\right)
$$

\subsubsection{Threshold Calculation:}

Suppose there exists $n$ distinct Gaussian clusters $C_{i}, i=$ $1, \ldots, n, \hat{H}$ must have $n$ peaks, denoted by $P(1), \ldots, P(n)$, and $n-1$ valleys, denoted as $V(1), \ldots, V(n-1)$. Then, the interval of $C_{i}$ in the smoothed histogram $\hat{H}$ will be $[V(i-1), V(i)-1]$, with $V(0)=1$ and $V(n)=G+1$. The optimal estimation interval within each cluster is defined to find the parameters which represent the distribution of cluster. Initial cluster mean value is determined by using a suitable interval whose length is given by $l_{i}=(1 / 2)(V(i)-V(i-1)$ 1 ). This length $l_{i}$, possesses the properties of $l_{i} \gg \sigma_{i}$ and $l_{i} \alpha \sigma_{i}$. The length of interval satisfies Tchebycheff inequality and can be used as the length of optimal parameter estimation interval.

Let $w$ be the searching window with length $l_{i}$ to search the location of optimal estimation interval of cluster $C_{i}$. The searching window $w$ which starts by placing the leftmost point at $V(i-1)$ slides toward the end of cluster $V(i)-1$ by moving one bin at a time. The searching process stops if the rightmost point reaches the end of cluster $V(i)-1$. There will be $V(i)-V(i-1)-1-l_{i}$ searching windows. Meanwhile, the skewness $S$ is calculated for each searching window $w_{j}, j=1,2, \ldots, V(i)-V(i-1)-1-l_{i}$, denoted by $S\left(w_{j}\right)$. The skewness is a measure of the asymmetry of the probability distribution of a real valued random variable. Skewness of each searching window for the bimodal histogram can be calculated as,

$$
\begin{gathered}
S\left(w_{j}\right)=\frac{\mu_{1}\left(w_{j}\right)}{\sqrt{\mu_{1}\left(w_{j}\right)}} \\
\mu_{n}\left(w_{j}\right)=\frac{\sum_{i \in w_{j}}\left(i-m_{i}\right)^{n} H(i)}{\sum_{i \in w_{j}} H(i)} \\
\tilde{m}_{w_{j}}=\frac{\sum_{i \in w_{j}} i H(i)}{\sum_{i \in w_{j}} H(i)}
\end{gathered}
$$

where, $\tilde{m}_{w_{j}}$ is the center of the searching window and $\mu_{n}\left(w_{j}\right)$ is the mean value of the windows between the clusters. $S\left(w_{j}\right)$ is the skewness of the searching window. The optimal interval $w_{o}$ to estimate the mean and variance of each cluster is found using minimum absolute skewness value, given by,

$$
w_{o}=\min \left|S_{1}\left(w_{j}\right)\right|
$$

The mean, variance and proportion of the cluster can then be determined by the following equations: The mean of a data set, and in particular of an image histogram, is the arithmetic average of the values in the set, obtained by summing all values and dividing by the number of them. The mean is, thus, a measure of the center of the distribution. 


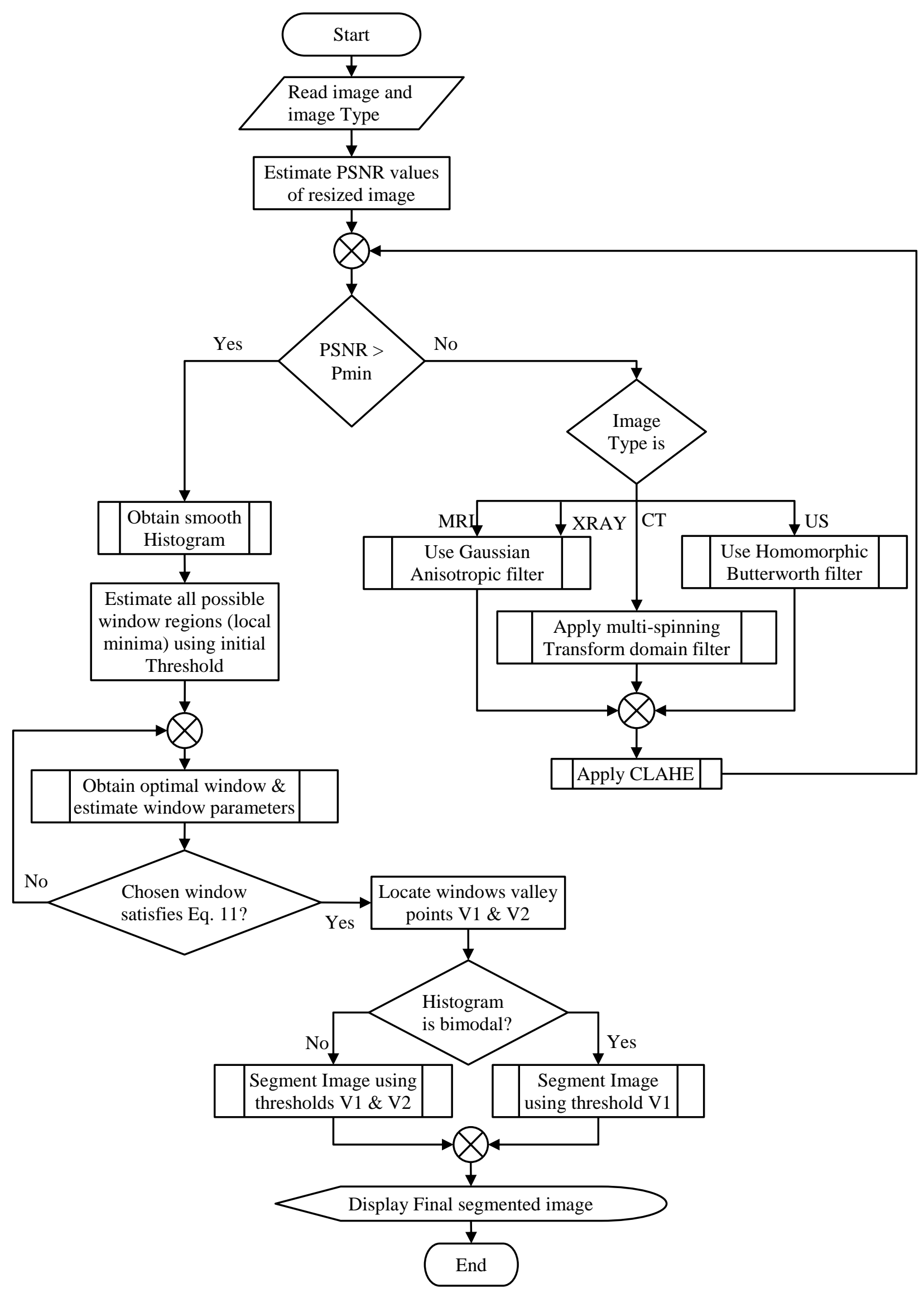

Fig.2. Flow diagram of proposed Algorithm 
The mean is a weighted average where the weight factors are the relative frequencies. The variance of a dataset is the arithmetic average of the squared differences between the values and the mean. The standard deviation is the squared root of the variance. The variance and the standard deviation are both measures of the spread of the distribution around the mean.

$$
\begin{gathered}
m_{i}=\frac{\sum_{i \in c_{i}} i H(i)}{\sum_{i \in c_{i}} H(i)} \\
\sigma_{i}^{2}=\frac{\sum_{i \in c_{i}}\left(i-m_{i}\right)^{2} H(i)}{\sum_{i \in c_{i}} H(i)} \text { and } p_{i}=\frac{\sum_{i \in c_{i}} H(i)}{\sum_{i=1}^{g} H(i)}
\end{gathered}
$$

$m_{i}$ is the mean value of the cluster, $\sigma_{i}^{2}$ is the cluster variance and $p_{i}$ is the cluster proportion. For the $i^{\text {th }}$ observation $H(i)$, it is more likely generated by cluster $C_{k}$ if,

$$
\frac{\hat{p}_{k}}{\sqrt{2 \pi \hat{\sigma}_{k}}} \exp \left\{-\frac{1}{2}\left(\frac{i-\hat{m}_{k}}{\hat{\sigma}_{k}}\right)^{2}\right\}>\frac{\hat{p}_{j}}{\sqrt{2 \pi \hat{\sigma}_{j}}} \exp \left\{-\frac{1}{2}\left(\frac{i-\hat{m}_{j}}{\hat{\sigma}_{j}}\right)^{2}\right\}
$$

For $1 \leq j \leq n, 1 \leq k \leq n$ and $j \neq k$.

If there are $n$ clusters, we will obtain $n$ - 1 threshold values $T_{i}, i$ $=1,2, \ldots, n-1$. Therefore, the $i^{\text {th }}$ threshold $T_{i}$ can be determined as follows:

$T_{i}=\max \left\{k: H(k)\right.$ is generated by the $i^{\text {th }}$ Gaussian cluster $\}$

Finally, for each cluster $C_{i}, i=1,2 \ldots, n$, the range becomes $[T(i-$ 1), $T(i)-1]$ with $T(0)=1$ and $T(n)=g$.

\section{PERFORMANCE MEASURES}

In the proposed approach, quality of the denoised images is measured using PSNR and the contrast of the enhanced image is estimated using $C N R$. Further, accuracy of segmented region is evaluated using sensitivity and specificity measures.

$$
\begin{gathered}
P S N R=10 \log _{10}\left[\frac{(\alpha-1)^{2}}{M S E}\right] d B \\
M S E=\frac{1}{M N} \sum_{i=0}^{M-1} \sum_{j=0}^{N-1}\left[I_{r}(i, j)-I_{e}(i, j)\right]^{2}
\end{gathered}
$$

where, $I_{r}$ is the input reference image and $I_{e}$ is the enhanced image. $M N$ is the size of the image and $\alpha$ is the dynamic range of image pixel values. For 8-bit image, $\alpha=256$. Contrast to Noise Ratio,

$$
\begin{gathered}
C N R=\frac{\mu_{r}-\mu_{e}}{\sigma_{n}} \\
\mu_{r}=\frac{1}{M N} \sum_{i=0}^{M-1} \sum_{j=0}^{N-1} I_{r}(i, j) \\
\mu_{e}=\frac{1}{M N} \sum_{i=0}^{M-1} \sum_{j=0}^{N-1} I_{e}(i, j) \\
\sigma_{n}=\sqrt{\frac{1}{M N-1} \sum_{i=0}^{M-1} \sum_{j=0}^{N-1}\left[I_{n}(i, j)-\mu_{n}\right]^{2}}
\end{gathered}
$$

where, $\mu_{r}$ is the mean of the reference image, $\mu_{e}$ is the mean value of the enhanced image, $\mu_{n}$ is the mean value of noisy image and $\sigma_{n}$ is the standard deviation of the noisy image.

Sensitivity or true positive rate is defined as the total number of pixels correctly detected as abnormal cells. It means test is positive.

$$
\text { Sensitivity }=\frac{N_{T P}}{N_{T P}+N_{T N}}
$$

Specificity or true negative rate is the percentage of pixels identified as normal region even though it is abnormal region. Specificity is a true negative measure that refers to the proportion of image containing a defective mass that has been incorrectly classified.

$$
\text { Specificity }=\frac{N_{T N}}{N_{T N}+N_{F N}}
$$

$N_{F N}$ is the number of pixels incorrectly classified as normal (non-tumor) region. $N_{T P}$ is the portion of the image correctly classified as defective mass. $N_{T N}$ is the portion of the image correctly classified as normal region.

\section{FLOW CHART OF THE PROPOSED METHODOLOGY}

The main goal of the proposed approach is to automatically find the optimal threshold value from the image histogram and to use this threshold value for accurate extraction of regions of interest from different modality medical images. Histogram decomposition is used to achieve precise threshold calculation. The Fig.2 shows the complete flow-diagram of the proposed approach.

\section{EXPERIMENTAL RESULTS}

The results are validated on an Intel(R) Core i5 CPU running at $2.40 \mathrm{GHz}$ with $8.00 \mathrm{~GB}$ of RAM. Software used for validation of the proposed approach is MATLAB R2012a on MS Windows 7, 64-bit operating environment. Experimental work is carried out on five types of medical image database, such as: MRI, CT, XRay, Ultrasound and Retinal imaging modalities. Each type consists of 10 images along with manually marked ground truth images. Each image is of size $256 \times 256$ with a lossless PNG file format.

In the proposed approach, an attempt is made to determine the optimal threshold value automatically using the information from image histograms. To avoid fake valley points and to obtain the smooth histogram, selected image is preprocessed initially. An image to be analyzed is chosen from the medical image database of different modalities. Type of noise varies from one modality to other. Hence based on the type of image selected (MRI, CT, US, $\mathrm{X}$-Ray and Eye), the algorithm switches to a particular preprocessing stage as indicated in the flow diagram Fig.2. In the preprocessing stage, the noise level is estimated for each input image using PSNR metric. Based on the type of noise, a suitable filter is used for noise reduction and the image quality improvement is again assessed. In addition, the contrast of the image is fine-tuned using CLAHE approach and its performance is evaluated using CNR metric. The parameters: PSNR and CNR 
are assessed for all the 10 images in each modality using the Eq.(15) and Eq.(16). However, denoised results are shown only for US fetus images, (Fig.3). Visual interpretations in Fig.3(a)Fig.3(d) reveal the qualitative improvement with respect to noise removal (Fig.3(c)) and contrast enhancement (Fig.3(d)). The quantitative assessment of the preprocessing stage given in Table.1. PSNR and CNR values for only two images in each category are indicated in the table. Readings in Table. 1 indicate the improvement in the quality of the pre-processed images. However, it can be seen from Table.1, there is an improvement in PSNR value for the denoised CT $(54.72 \mathrm{~dB}$ to $58.72 \mathrm{~dB})$ and $\mathrm{X}-$ Ray (48.72dB to $51.22 \mathrm{~dB}$ ) images. But, PSNR values of (MRI: $34.72 \mathrm{~dB}$ to $34.72 \mathrm{~dB}$, US: $29.56 \mathrm{~dB}$ to $28.95 \mathrm{~dB}$ and Eye images: $28.02 \mathrm{~dB}$ to $25.21 \mathrm{~dB}$ ) are comparatively less. Further it can be noticed that after subjecting these images to enhancement process, improvement in PSNR values demonstrates the need for CLAHE for poor contrast medical images such as MRI, US and Eye images. It can be noticed that the CNR values for all the enhanced medical images is more compared to denoised images. This contrast improvement is necessary for accurate ROI extraction. Once the images are preprocessed, their histograms are obtained and smoothed to find the threshold values using the statistical moments of the image histograms. The Table. 3 shows the sample histograms of five preprocessed images considering one image in each modality along with manually labeled images and segmented output by the proposed approach. The estimated parameters (mean, variance and proportions) and the corresponding threshold value for only one medical image i,e. Ultrasound image whose histogram has two clusters are tabulated in Table.2 and the parameters calculated for rest of the medical images are not shown. Using these statistical parameters, skewness is determined. Suppose if the histogram has two equal peaks, then the valley with highest skewness gives the optimal threshold value. Image histogram with one cluster has a single threshold value and histograms with three clusters have two threshold values which can be seen in Table.2. In general $n$ clustered histogram has $n-1$ threshold values. Finally, optimal threshold value is determined and the same is used to segment the image.

Table.1. Experimental results of preprocessing stage

\begin{tabular}{|c|c|c|c|c|}
\hline Metric & $\begin{array}{l}\text { Image } \\
\text { type }\end{array}$ & $\begin{array}{l}\text { Input } \\
\text { image }\end{array}$ & $\begin{array}{c}\text { Denoised } \\
\text { image }\end{array}$ & $\begin{array}{c}\text { Enhanced image } \\
\text { using CLAHE }\end{array}$ \\
\hline \multirow{10}{*}{$\begin{array}{l}\text { PSNR } \\
\text { (Db.) }\end{array}$} & MRI1 & 34.72 & 34.72 & 37.48 \\
\hline & MRI2 & 35.212 & 35.223 & 35.848 \\
\hline & CT1 & 54.72 & 58.72 & 59.48 \\
\hline & CT2 & 48.72 & 51.22 & 52.48 \\
\hline & X-Ray1 & 48.72 & 51.22 & 52.48 \\
\hline & X- Ray2 & 42.18 & 44.12 & 49.56 \\
\hline & US1 & 25.12 & 24.52 & 32.51 \\
\hline & US2 & 29.56 & 28.95 & 36.02 \\
\hline & Eye1 & 28.02 & 25.21 & 30.18 \\
\hline & Eye2 & 35.12 & 34.52 & 38.45 \\
\hline \multirow{3}{*}{ CNR } & MRI1 & 24.03 & 24.06 & 47.92 \\
\hline & MRI2 & 24.06 & 24.06 & 30.18 \\
\hline & CT1 & 24.03 & 24.06 & 47.92 \\
\hline
\end{tabular}

\begin{tabular}{|c|c|c|c|c|}
\hline \multirow{4}{*}{ CT2 } & 25.03 & 24.66 & 37.93 \\
\cline { 2 - 5 } & X-Ray1 & 25.03 & 24.06 & 37.93 \\
\cline { 2 - 5 } & X- Ray2 & 25.13 & 24.66 & 35.29 \\
\cline { 2 - 5 } & US1 & 16.53 & 18.26 & 26.81 \\
\hline & US2 & 19.15 & 20.06 & 40.29 \\
\hline & Eye1 & 25.03 & 24.06 & 32.12 \\
\cline { 2 - 5 } & Eye2 & 26.03 & 28.06 & 36.48 \\
\hline
\end{tabular}

Table.2. Statistical parameters for threshold estimation

\begin{tabular}{|c|l|c|}
\hline \multicolumn{1}{|c|}{ Images } & \multicolumn{2}{|c|}{ Statistical Parameters } \\
\hline \multirow{4}{*}{$\begin{array}{c}\text { Ultrasound image } \\
\text { Two clusters }\end{array}$} & Mean1 & 87 \\
\cline { 2 - 3 } & Variance1 & 324 \\
\cline { 2 - 3 } & Proportion1 & 0.27 \\
\cline { 2 - 3 } & Mean2 & 133 \\
\cline { 2 - 3 } & Variance2 & 142 \\
\cline { 2 - 3 } & Proportion2 & 0.32 \\
\cline { 2 - 3 } & Threshold & 52 \\
\hline
\end{tabular}

In the proposed approach, ten images in each type are used for segmentation process. However, Fig.3 demonstrates the segmentation results for only one image to draw the visual inference. The accuracy of the segmentation results of the proposed approach is compared with other existing techniques such as: Otsu's thresholding, Region growing, image matting techniques: Alpha matting, Bayesian matting and Poisson matting. The quantitative analysis of the segmentation is demonstrated in Table.4.

Table.3. Table showing five sample images with their histograms, ground truth and segmented output

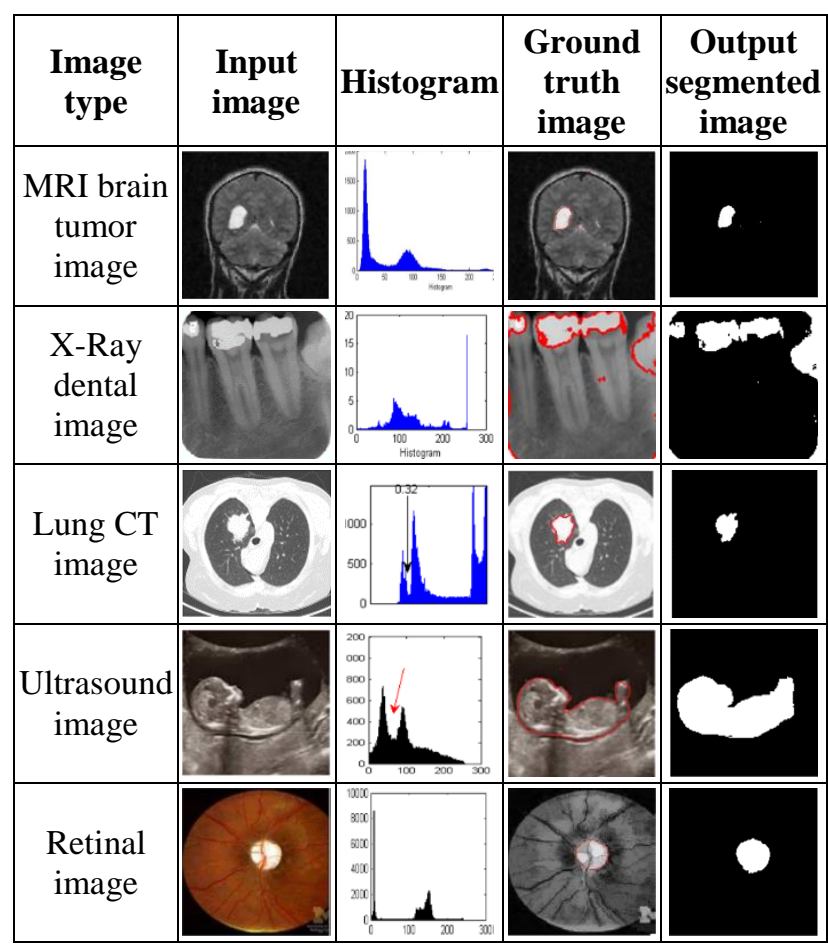

The Fig. 3 shows denoising and segmentation results of US fetus image. The Fig.3(a)-Fig.3(d) are preprocessed images. The 
Fig.3(e) is manually labeled image. Preprocessed image is segmented using Otsu's thresholding, region growing, image matting techniques and the proposed approach. Otsu's technique is simple to design and execution time is less $(13 \mu \mathrm{S}$ for US image) compared to other techniques as indicated in Table.4. However, it can be noticed in Fig.3(f), Otsu's technique has produced poor segmentation results (only $77 \%$ accuracy) and the image is distorted, whereas region growing technique, (Fig.3(g)) produces good segmentation accuracy of $98.5 \%$ and algorithmic time complexity is also moderate. But this method needs an initial parameter selection, such as the seed point. Improper seed point selection leads to poor segmentation results. On the other hand, three image matting techniques also result in appreciable accuracy. Among three techniques, Bayesian matting, (Fig.3(j)) and Poisson matting, (Fig.3(k)) techniques show the improvement in accuracy (98.66\% and 98.39\%) and less execution time (35.10

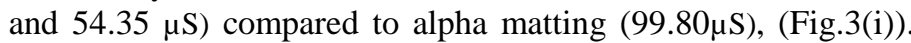
However, the main drawback of matting techniques is that they need 'trimap' as in Fig.3(h) which is to be specified by the user before segmentation process. This means all these methods demand human intervention in one way or the other and thereby prone to human errors and affect the segmentation accuracy. Whereas in the proposed approach, the parameters are automatically found using the statistical information of image histograms. It is evident from the results that the segmentation accuracy of the proposed method is relatively better $(98.71 \%$ for US image) compared to other techniques as recorded in Table. 4

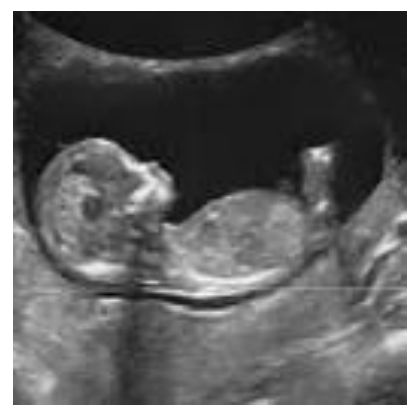

(a) Original input image

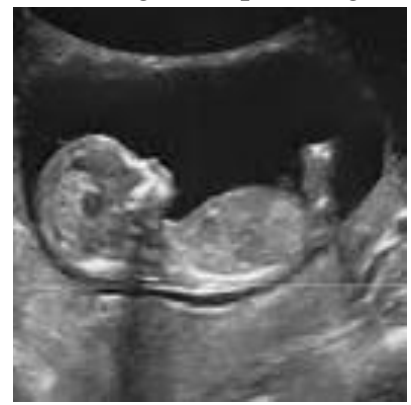

(c) Denoised image

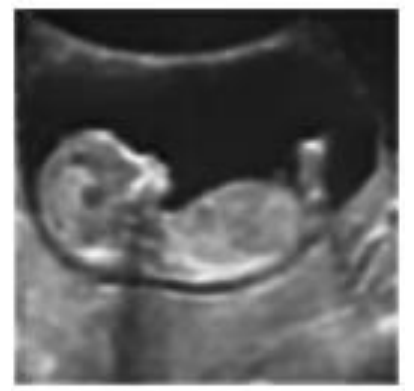

(b) Noisy image

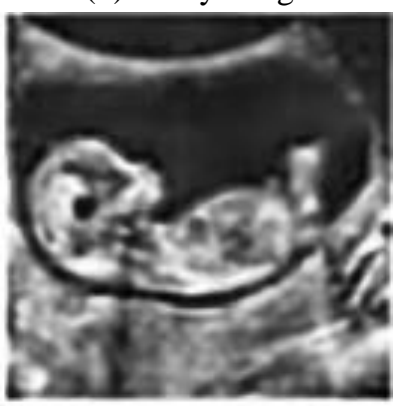

(d) Enhanced image

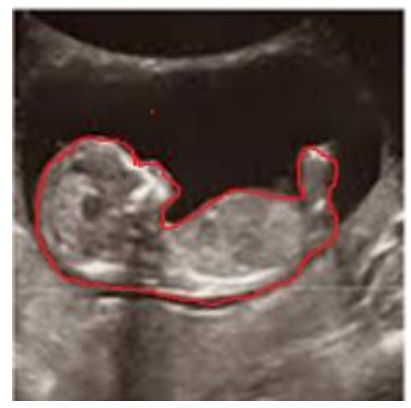

(e) Ground truth image

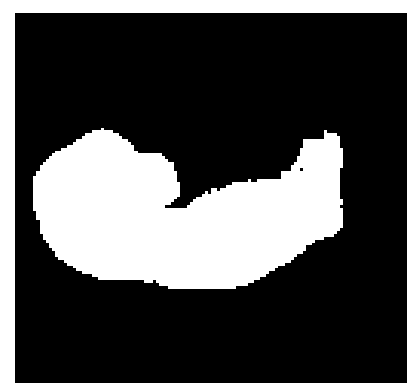

(g) Region Growing

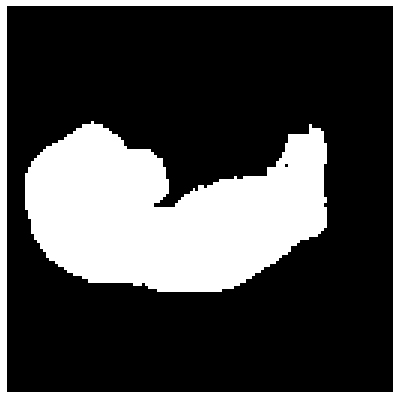

(i) Alpha matting

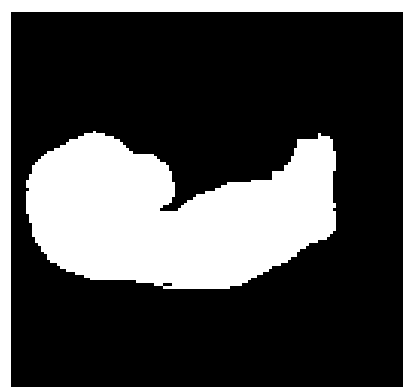

(k) Poisson matting

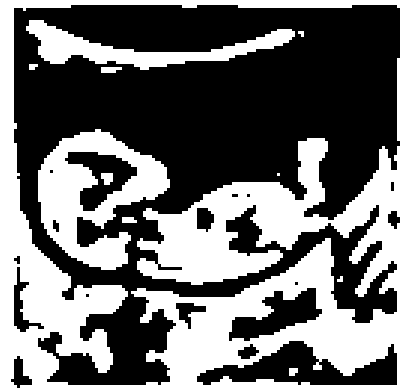

(f) Otsu's

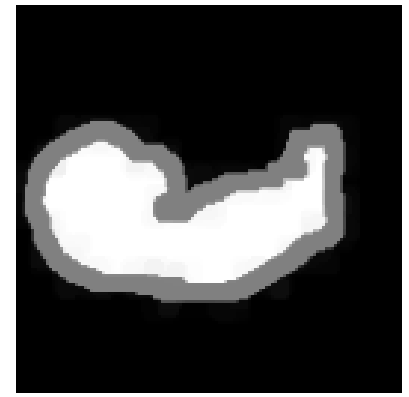

(h) Trimap

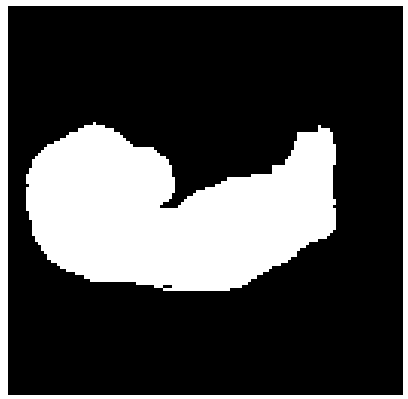

(j) Bayesian matting

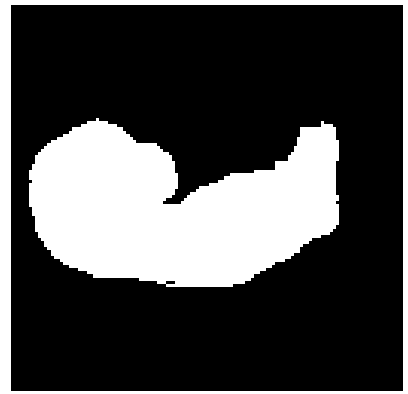

(1) histogram
Fig.3. Qualitative Analysis of Denoising and ROI Extraction Process for US image

Table.4. Quantitative Analysis of ROI Extraction

\begin{tabular}{|c|l|c|c|c|c|}
\hline \multirow{2}{*}{ Image type } & $\begin{array}{c}\text { Image segmentation } \\
\text { method }\end{array}$ & $\begin{array}{c}\text { Sensitivity } \\
\boldsymbol{S =} \frac{\boldsymbol{T P}}{\boldsymbol{T P +}+\boldsymbol{F N}}\end{array}$ & $\begin{array}{c}\text { Specificity } \\
\boldsymbol{G}=\frac{\boldsymbol{T N}}{\boldsymbol{T N}+\boldsymbol{F P}} \boldsymbol{\%}\end{array}$ & $\begin{array}{c}\text { Accuracy } \\
=\frac{\boldsymbol{S}+\boldsymbol{G}}{\boldsymbol{2}} \boldsymbol{\%}\end{array}$ & $\begin{array}{c}\text { Execution } \\
\text { time }(\boldsymbol{\mu S})\end{array}$ \\
\hline \multirow{3}{*}{ MRI } & Otsu's threshold & 80.00 & 83.33 & 81.66 & 10.05 \\
\cline { 2 - 6 } & Region growing & 98.00 & 98.80 & 98.40 & 40.40 \\
\cline { 2 - 6 } & Alpha matte & 96.33 & 97.00 & 96.66 & 100.80 \\
\cline { 2 - 6 } & Bayesian matte & 99.00 & 98.33 & 98.665 & 36.10 \\
\hline
\end{tabular}




\begin{tabular}{|c|c|c|c|c|c|}
\hline & Poisson matte & 98.80 & 98.33 & 98.565 & 50.35 \\
\hline & Histogram threshold & 99.00 & 98.33 & 98.66 & 33.55 \\
\hline \multirow{6}{*}{ X-Ray } & Otsu's threshold & 83.00 & 84.00 & 83.50 & 11.05 \\
\hline & Region growing & 98.00 & 98.33 & 98.15 & 42.40 \\
\hline & Alpha matte & 97.23 & 96.33 & 96.78 & 99.80 \\
\hline & Bayesian matte & 99.00 & 98.23 & 98.61 & 35.10 \\
\hline & Poisson matte & 99.00 & 98.00 & 98.50 & 54.35 \\
\hline & Histogram threshold & 99.00 & 99.30 & 99.15 & 30.55 \\
\hline \multirow{6}{*}{ Retinal } & Otsu's threshold & 83.33 & 85.00 & 84.165 & 11.05 \\
\hline & Region growing & 98.00 & 98.50 & 98.25 & 8.40 \\
\hline & Alpha matte & 98.10 & 98.23 & 98.16 & 99.80 \\
\hline & Bayesian matte & 98.99 & 98.56 & 98.77 & 67.10 \\
\hline & Poisson matte & 99.12 & 98.45 & 98.78 & 54.35 \\
\hline & Histogram threshold & 99.15 & 98.00 & 98.57 & 30.55 \\
\hline \multirow{6}{*}{$\mathrm{CT}$} & Otsu's threshold & 80.00 & 83.33 & 81.66 & 11.05 \\
\hline & Region growing & 99.00 & 99.13 & 99.06 & 34.40 \\
\hline & Alpha matte & 98.33 & 97.00 & 97.66 & 106.80 \\
\hline & Bayesian matte & 98.76 & 96.33 & 97.54 & 67.10 \\
\hline & Poisson matte & 98.54 & 98.33 & 98.43 & 50.35 \\
\hline & Histogram threshold & 99.00 & 98.78 & 98.89 & 30.55 \\
\hline \multirow{6}{*}{ US } & Otsu's threshold & 78.00 & 77.42 & 77.71 & 13.05 \\
\hline & Region growing & 99.00 & 98.00 & 98.5 & 32.40 \\
\hline & Alpha matte & 96.00 & 96.78 & 96.39 & 116.80 \\
\hline & Bayesian matte & 98.00 & 99.33 & 98.66 & 67.10 \\
\hline & Poisson matte & 98.78 & 98.00 & 98.39 & 50.35 \\
\hline & Histogram threshold & 99.00 & 98.43 & 98.71 & 23.55 \\
\hline
\end{tabular}

\section{CONCLUSION}

In this paper, an automatic region detection technique has been proposed for extracting desired regions from noisy medical images of all modalities. The proposed method attempted to find an optimal threshold value automatically using the parameters such as: mean, variance and skewness by decomposing the histogram into non-overlapping distributions employing histogram smoothing. The predefined moments estimated for each distribution in the histogram reduces the computational complexity compared to other conventional methods which usually suffer from iterative initial parameter refinement. Performance of the proposed algorithm is tested on user defined medical image database of all modalities and compared with other techniques. Experimental results demonstrate relative improvement in segmentation accuracy compared to other techniques. The proposed approach works well for the user defined dataset. For medical images from unknown database, the algorithm finds it difficult to identify the image type and to model denoising filter by itself.

\section{REFERENCES}

[1] D.J. Withey and Z.J. Koles, "Three Generations of Medical Image Segmentation: Methods and Available Software",
International Journal of Bioelectromagnetism, Vol. 9, No. 2, pp. 67-68, 2007.

[2] Dzung L. Pham, Chenyang Xu and Jerry L. Prince, "Current methods in Medical Image Segmentation", Annual Review of Biomedical Engineering, Vol. 2, pp. 315-337, 2000.

[3] Sanjay Agrawal, Rutuparna Panda, Sudipta Bhuyan and B.K. Panigrahi, "Tsallis Entropy based Optimal Multilevel Thresholding using Cuckoo Search Algorithm”, Swarm and Evolutionary Computation, Vol. 11, pp. 16-30, 2013.

[4] Jan H Waarsing, Judd S Day and Harrie Weinans, "An Improved Segmentation method for in Vivo Micro CT Imaging", Journal of Bone and Mineral. Research, Vol. 19, pp. 1640-1650, 2004.

[5] Preeti Gupta, Vandana Malik and Mallika Gandhi, "Implementation of Multilevel- Thresholding Technique for Medical Images", International Journal of Advanced Research in Computer Science and Software Engineering, Vol. 2, No. 2, pp. 1-3, 2012.

[6] M. Sezgin and B. Sankar, "Survey Over Image Thresholding Techniques and Quantitative Performance Evaluation", Journal of Electronic Imaging, Vol. 13, No. 1, pp. 146-165, 2004.

[7] Guang Yang, Kexiong Chen, Maiyu Zhou, ZhonglinXu and Yongtian Chen., "Study on Statistics Iterative Thresholding Segmentation based on Aviation Image", Proceedings of $8^{\text {th }}$ 
ACIS International Conference on Software Engineering, Artificial Intelligence, Networking, and Parallel/Distributed Computing, pp. 187-188, 2007.

[8] S. Gopinathan and P. Deepa, "Enhancement of Image Segmentation using Automatic Histogram Thresholding", International Journal on Recent and Innovation Trends in Computing and Communication, Vol. 3, No. 4, pp. 18631872, 2015.

[9] P.K. Sahoo S. Soltani and A.K.C. Wong, "A Survey of Thresholding Techniques", Computer Vision, Graphics, and Image Processing, Vol. 41, pp. 233-260, 1988.

[10] P. Daniel Ratna Raju and G. Neelima, "Image Segmentation by using Histogram Thresholding", International Journal of Computer Science Engineering and Technology, Vol. 2, No. 1, pp. 776-779, 2012.

[11] Lois Hertz and Ronald W. Schafer, "Multilevel Thresholding using Edge Matching", Computer Vision, Graphics and Image Processing, Vol. 45, No. 3, pp. 279295, 1998.

[12] Seokwon Yeom, "Multi-Level Segmentation of Infrared Images with Region of Interest Extraction", International Journal of Fuzzy Logic and Intelligent Systems, Vol. 16, No. 4, pp. 246-253, 2016

[13] S Pal and P. Bhattacharya, "Multipeak Histogram Analysis in Region Splitting: A Regularization Problem", IEE Proceedings E - Computers and Digital Techniques, Vol. 138, No. 4, pp. 285-288, 1991.

[14] Mark J. Carletto, "Histogram Analysis using Scale space Approach", IEEE Transactions, Pattern Analysis Machine Intelligence, Vol. 9, No. 1, pp. 121-129, 1981.

[15] Thresiamma Devasia, Poulose Jacob and Tessamma Thomas, "Automatic Extraction and Localisation of Optic Disc in Colour Fundus Images", International Journal of Computer Science Issues, Vol. 12, No. 3, pp. 146-154, 2015.

[16] X. Zhuang, T. Wang and P. Zhang, "A Highly Robust Estimator through Partially likelihood Function Modeling and its Application in Computer Vision", IEEE Transactions Pattern Analysis. Machine Intelligence, Vol. 14, No. 1, pp. 19-35, 1992.

[17] C.C. Chang and L.L. Wang., "A Fast Multilevel Thresholding Method Based on Lowpass and Highpass Filtering", Pattern Recognition Letters, Vol. 18, No. 14, pp. 1469-1478, 1997.
[18] Yang Wang and Haomin Zhou, "Total Variation Wavelet Based Medical Image Denoising", International Journal of Biomedical Imaging, Vol. 2006, pp. 1-6, 2006.

[19] Albert Macovski, "Magnetic Resonance in Medicine", Magnetic Resonance in Medicine, Vol. 36, No. 3, pp. 494497, 1996.

[20] Isshaa Aarya, Danchi Jiang and Timothy Gale, "Signal Dependent Rician Noise Denoising using Nonlinear Filter", Lecture Notes on Software Engineering, Vol. 1, No. 4, pp. 344-349, 2013.

[21] Henri H. Arsenault and M. Denis, "Image Processing in Signal-Dependent Noise", Canadian Journal of Physics, Vol. 61, No. 2, pp. 309-317, 1983.

[22] Hensel M, Brummund U, Pralow T, and Grigat R.R, "Pyramid Multiscale Products for Noise Reduction of Medical X-Ray Image Sequences", Biomedical Engineering/Biomedizinische Technik, Vol. 50-1, pp. 11081109, 2005.

[23] Flourian Luiser, "Image Denoising in Mixed PoissonnGaussian Noise", IEEE Transactions on Image Processing, Vol. 20, No. 3, pp. 696-708, 2011.

[24] B.N. Aravind and K.V. Suresh., "Multispinning for Image Denoising”, International Journal for Intelligent Systems, Vol. 21, No. 3, pp. 271-291, 2012.

[25] Edwin Hewitt and Robert E. Hewitt, "The Gibbs-Wilbraham Phenomenon: An Episode in Fourier Analysis", Archive for History of Exact Sciences, Vol. 21, No. 2, pp. 129-160, 1979.

[26] A. Mohanapreethi and V. SrinivasaRaghavan, "Performance Evaluation of Various Filtering Techniques for Speckle Suppression in Ultrasound Images", International Journal of Research in Advent Technology, Vol. 2, No. 4, pp. 29-35, 2014

[27] Jeng Horng Chang, Kuo Chin Fan and Yang-Lang Chang, "Multilevel Gray level Histogram Modeling and Decomposition", Image and Vision Computing, Vol. 20, pp. 203-216, 2002

[28] A.M. Reza, "Realization of the Contrast Limited Adaptive Histogram Equalization for Real-Time Image Enhancement", Journal of VLSI Signal Processing systems for Signal, Image and Video Technology, Vol. 38, No. 1, pp. 35-44, 2004. 